
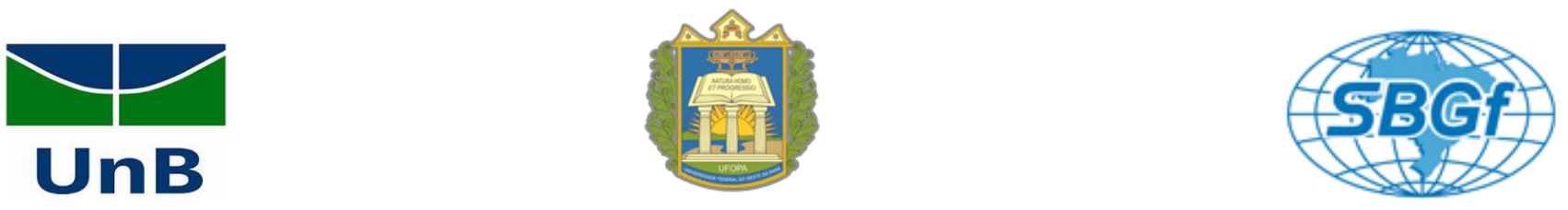

\title{
Estudo preliminar da subducção da placa de Nazca sob a placa sul-americana no norte do Brasil através de tomografia sísmica de tempo de percurso com ondas $\mathbf{P}$
}

\author{
Sebastião Wendell Nobres Moura, Universidade Federal do Oeste do Pará (UFOPA) \\ Paulo Araújo de Azevedo, Universidade Federal do Oeste do Pará (UFOPA) \\ Marcelo Peres Rocha, Universidade de Brasília (UnB)
}

Copyright 2018, SBGf - Sociedade Brasileira de Geofísica

Este texto foi preparado para a apresentação no VIII Simpósio Brasileiro de Geofísica, Salinópolis, 18 a 20 de setembro de 2018. Seu conteúdo foi revisado pelo Comitê Sécnico do VIII SimBGf, mas não necessariamente representa a opinião da SBGf ou de seus associados. É proibida a reprodução total ou parcial deste material para propósitos comerciais sem prévia autorização da SBGf.

\section{Resumo}

Neste trabalho, apresentamos resultados de tomografia sísmica de tempo de percurso utilizando ondas $P$ no Brasil. Foram realizadas inversões para dados registrados durante o período de 1992-2015 por 264 estações. O objetivo deste trabalho foi fazer uma proposta da localização da placa subduzida de Nazca sob a placa sul-americana. Anomalias de alta velocidade sob a bacia Amazônica sugerem a presença da placa de Nazca nesta região.

\section{Introdução}

A formação tectônica da América do Sul (Figura 1) está relacionada principalmente ao cinturão andino a oeste, à plataforma sul-americana em sua porção central e a plataforma da Patagônia ao sul (Ramos, 1999; Almeida et al., 2000). Às margens do continente sul-americano há sistemas complexos de placas tectônicas. Neste contexto, a oeste deste continente localiza-se a Placa oceânica de Nazca, formando uma zona de convergência com a placa Sul-Americana, responsável por terremotos nos países localizados a oeste da América do Sul, como por exemplo, o Chile e Peru e outros. O que se conhece a respeito destas placas, deve-se principalmente aos estudos sismológicos. Parte das informações a respeito da subducção da placa de Nazca sob a placa sulamericana foi obtida através de métodos de tomografia sísmica (e.g. Schimmel et al., 2003; Rocha et al., 2011). Os modelos tomográficos podem revelar informações sobre a estrutura litosférica e do manto em grandes profundidades onde não há informações disponíveis sobre a sismicidade. O objetivo deste trabalho é fazer uma proposta da localização da placa subduzida de Nazca sob a placa sul-americana utilizando o método de tomografia sísmica de tempo de percurso com ondas $P$ (VanDecar et al., 1990) como referência, visando dar futuras contribuições ao entendimento da evolução tectônica no continente sul-americano e as relações desta subducção com a sismicidade no norte do Brasil. A área de estudo deste trabalho está representada pelo quadrilátero tracejado na Figura 1. Os resultados relativos as demais regiões mostradas na Figura 1 foram discutidas em outros trabalhos (Rocha et al., 2011; Azevedo et al., 2011; Rocha et al., 2016).

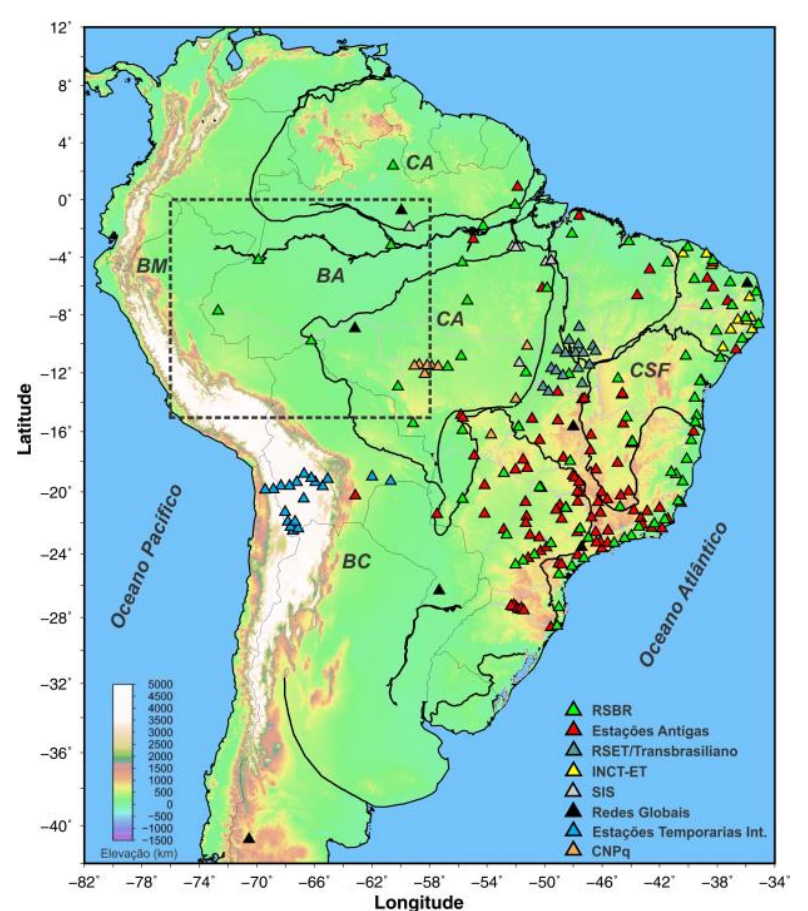

Figura 1: Área de estudo (quadrilátero tracejado) com a distribuição das estações sismográficas. BM=Bacia Maranon; $\mathrm{BA}=$ Bacia Amazônica; $\mathrm{CA}=$ Cráton Amazônico; $\mathrm{BC}=\mathrm{Bacia}$ do Chaco; CSF=Cráton do São Francisco.

\section{Metodologia/ Problema Investigado}

Os dados utilizados neste trabalho são de estações sismográficas instaladas principalmente no Brasil (Figura 1), especialmente estações pertencentes à Rede Sismográfica Brasileira (RSBR) e de acesso público. Utilizamos as fases $P$ e PKIKP para eventos telessísmicos com magnitude mínima de 4,6 e 5,4, respectivamente. Estes valores de magnitude permitem observar chegadas mais claras nos sismogramas. Foram utilizadas distâncias epicentrais entre $30^{\circ}$ e $95^{\circ}$ para ondas $\mathrm{P}$ e $150^{\circ}$ e $180^{\circ}$ para PKIKP o que permite evitar efeitos de triplicação de fase causados pela zona de transição (Press et al., 2006). Utilizamos um total de 43211 chegadas e 4692 eventos. A Figura 2 mostra a distribuição epicentral dos eventos utilizados. Os dados aqui utilizados são resíduos de tempo de chegadas de ondas geradas por terremotos distantes (telessísmos), os quais são convertidos em perturbações laterais de velocidades (Evans e Achauer 1993). Os tempos 
observados foram obtidos pela marcação das fases nos sismogramas, e os tempos teóricos foram calculados a partir de um modelo de referência da Terra (IASP91 Kennett e Engdahl, 1991). A tomografia sísmica utilizado neste trabalho segue a formulação empregada pelo método ACH (Evans e Achauer, 1993), onde é possível restringir as anomalias de velocidade ao seguimento final dos raios sísmicos, eliminando influências de estruturas fora do volume estudado.

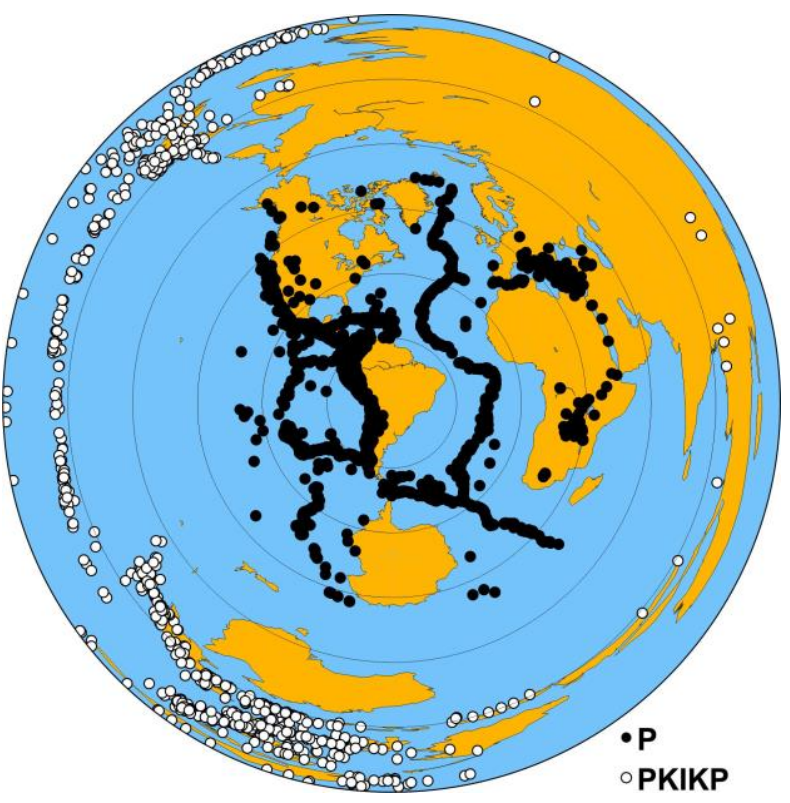

Figura 2: Distribuição epicentral dos eventos utilizados neste trabalho. Os círculos concêntricos marcam intervalos de distâncias a cada $30^{\circ}$.

\section{Resultados}

As Figuras 3 e 4 apresentam os resultados obtidos. Algumas das anomalias de velocidade que aparecem nestas figuras já foram descritas nos trabalhos anteriores. Em nossos resultados, as perturbações de velocidades resultantes, a partir do modelo médio de velocidades (IASP91 - Kenneth e Engdahl, 1991), são apresentadas no mapa horizontal (Figuras 3) e no perfil vertical (Figura 4), o qual se estendeu para além da área de estudo para uma melhor interpretação. As anomalias representam heterogeneidades laterais do modelo de ondas $P$, onde as cores frias representam anomalias de alta velocidade e cores quentes representam anomalias de baixa velocidade. As regiões com baixa densidade de raios (menos de 20 raios $/ 100 \mathrm{~km}^{3}$ ) são mostradas em preto. As estruturas são interpretadas a partir de profundidades mantélicas, pois não há resolução para estruturas da crosta terrestre, uma vez que os raios sísmicos chegam verticalizados e não há cruzamento entre eles na parte superior do modelo. Dessa forma, os primeiros $50 \mathrm{~km}$ de profundidade foram ocultados nos resultados. Anomalias de alta velocidade foram observadas em toda porção mapeada do Cráton Amazônico. As maiores velocidades estendem-se sob a região da bacia Amazônica.
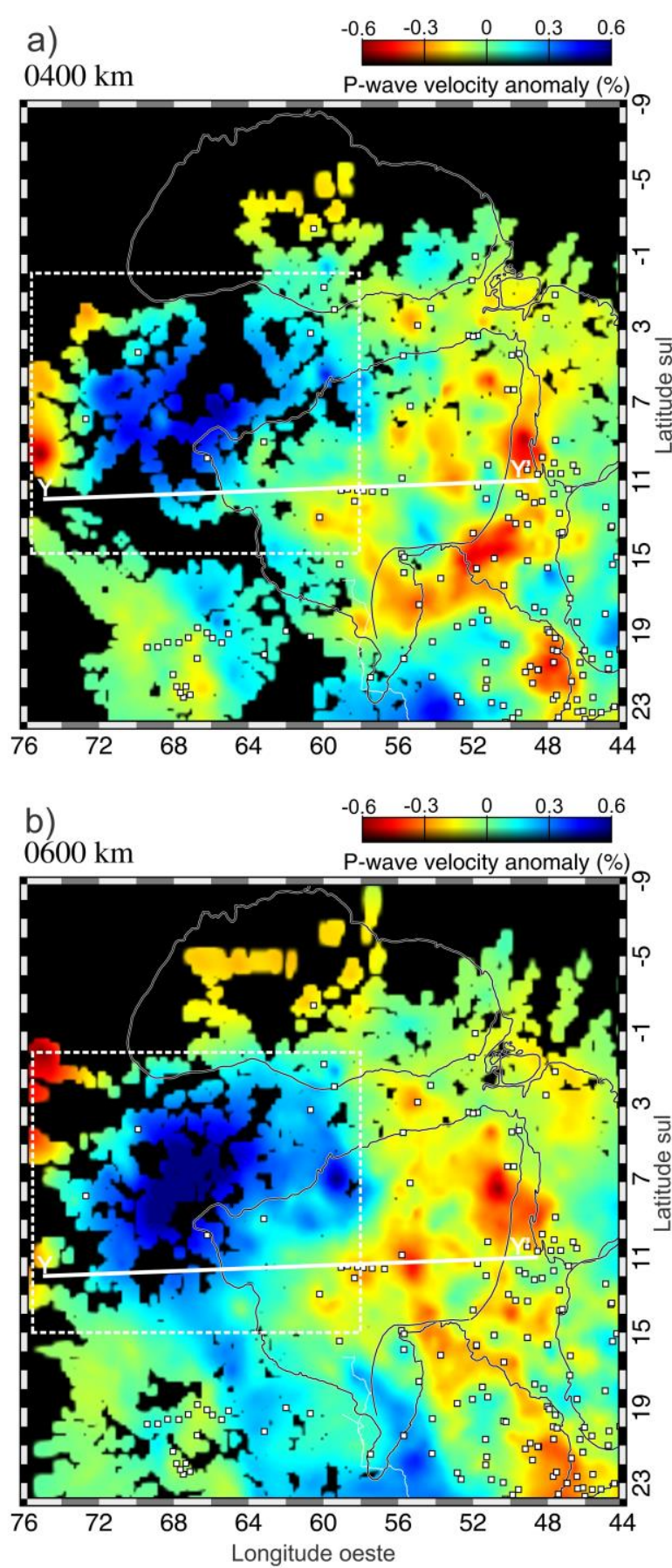

Figura 3: Imagem tomográfica horizontal para a profundidade de $400 \mathrm{~km}$ (a) e $600 \mathrm{~km}$ (b). O quadrilátero tracejado representa a localização da área de estudo. 


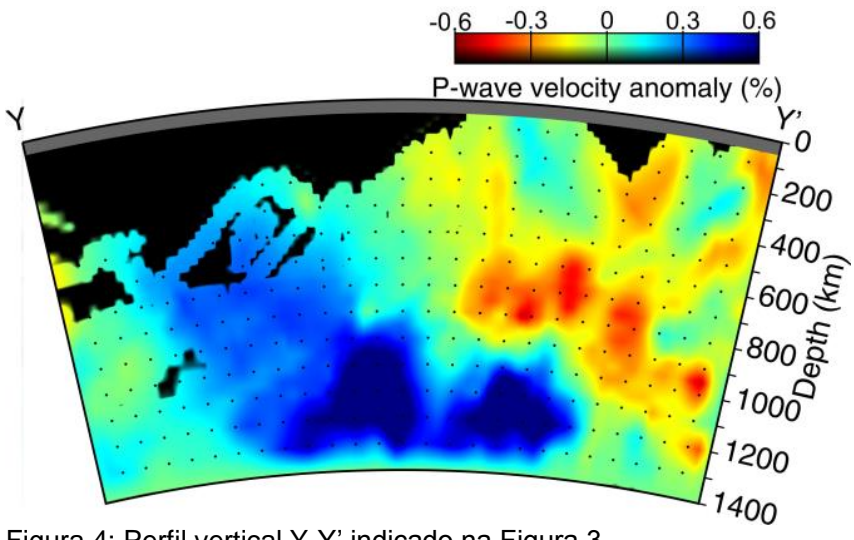

Figura 4: Perfil vertical $Y-Y^{\prime}$ indicado na Figura 3.

\section{Discussão e Conclusões}

A anomalia de alta velocidade observada na área de estudo foi interpretada como resultado da subducção da placa de Nazca sob a placa sul-americana em profundidades maiores que $400 \mathrm{~km}$. Observa-se na Figura 4 que há uma tendência de mergulho diagonal das anomalias de alta velocidade entre as latitudes 57-71 graus oeste ao longo do perfil $Y$-Y'. Na profundidade de $1400 \mathrm{~km}$ a anomalia torna-se horizontalizada devido à profundidade do modelo de parâmetros que foi utilizada (Azevedo, 2017). A subducção da placa de Nazca perto desta região em latitudes superiores a 71 graus oeste é relativamente plana (e.g. Espurt et al., 2007), devido à subducção da dorsal de Nazca sob a placa de Nazca (e.g. Gutscher et al., 2000). Para continuação dos trabalhos esperamos incluir outras estações sismográficas na região amazônica, de modo a melhorar a resolução das anomalias tomográficas, e também realizar testes de resolução para dar maior credibilidade aos resultados e aprofundar mais as discussões.

\section{Agradecimentos}

Ao pesquisador Dr. John VanDecar, pelos programas disponibilizados para a realização da tomografia utilizada neste trabalho. Ao Observatório Sismológico da Universidade de Brasília (SIS/UnB), pelos dados de algumas estações cedidos a este trabalho. Ao CNPQ pelo financiamento dos projetos de pesquisa "Estudos Tectônicos sobre a Bacia dos Parecis" e "Estudo da estrutura sísmica da Crosta e do Manto Superior nas porções sul e sudeste do limite entre a Província Tocantins e o cráton Amazônico", que também cederam dados de estações sismográficas utilizadas neste trabalho. A Rede Sismográfica Brasileira, pela disponibilização dos dados da maior parte das estações utilizadas.

\section{Referências}

Almeida, F. F. M.; Neves, B. B. B., e Carneiro, C. D. R. (2000). The origin and evolution of the South American platform. Earth Science Reviews, 50(1-2):77-111.

Azevedo, P. A. 2017. Estudo do manto superior sob o Brasil utilizando tomografia sísmica de tempo de percurso com onda P., Universidade de Brasília, Instituto de Geociências, Tese de Doutorado.

Espurt, N.; Baby, P.; Brusset, S.; Roddaz, M.; Hermoza, W.; Regard, V.; Antoine, P. O.; Salas-Gismondi, R. and Bolaños, R. (2007). How does the Nazca Ridge subduction infl uence the modern Amazonian foreland basin? Geology, v.35; no. 6; p. 515-518; doi:10.1130/G23237A.1.

Evans, J. R. e Achauer, U. (1993). Teleseismic velocity tomography using the $\mathrm{ACH}$ method: theory and application to continental-scale studies, In: H. M. Iyer e K. Hirahara eds. Seismic Tomography: Theory and Practice: Chapman and Hall, London, pp. 319-360.

Gutscher, M.-A., Spakman, W., Bijwaard, H., and Engdalh, E.R., 2000, Geodynamics of flat subduction: Seismicity and tomographic constraints from the Andean marin: Tectonic v.19, p.814-833 doi:10.1029/1999TC001152.

Kenneth, B. L. N. e Engdahl, E. R. (1991). Travel times for global earthquakes location and phase identification. Geophysical Journal International, 105:429-465.c

Press, F.; Silver, R.; Grotzinger, J. e Jordan, T. (2006). Para entender a Terra. 4 ed. cap. 20.

Ramos, V. A. (1999). Plate tectonic setting of the Andean Cordillera. Episodes,22(3):183-190.

Rocha, M. P.; Schimmel, M. e Assumpção, M. (2011). Upper-mantle seismic structure beneath SE and Central Brazil from $\mathrm{P}_{-}$and S-wave regional travel time tomography. Geophysical Journal International, 184:268286, doi: 10.1111/j.1365-246X.2010.04831.x.

VanDecar, J. C. e R. S. Crosson, (1990). Determination of teleseismic relative phase arrival times using multichannel cross-correlation and least squares, Bull.Seismology Soc. Am., 80, 150-169. 